\title{
Bayesian decision theoretic two-stage design in phase II clinical trials with survival endpoint
}

\author{
Lili Zhao, ${ }^{a * \dagger}$ Jeremy M. G. Taylor $^{\mathrm{b}}$ and Scott M. Schuetze ${ }^{\mathrm{c}}$
}

\begin{abstract}
In this paper, we consider two-stage designs with failure-time endpoints in single-arm phase II trials. We propose designs in which stopping rules are constructed by comparing the Bayes risk of stopping at stage I with the expected Bayes risk of continuing to stage II using both the observed data in stage I and the predicted survival data in stage II. Terminal decision rules are constructed by comparing the posterior expected loss of a rejection decision versus an acceptance decision. Simple threshold loss functions are applied to time-to-event data modeled either parametrically or nonparametrically, and the cost parameters in the loss structure are calibrated to obtain desired type I error and power. We ran simulation studies to evaluate design properties including types I and II errors, probability of early stopping, expected sample size, and expected trial duration and compared them with the Simon two-stage designs and a design, which is an extension of the Simon's designs with time-to-event endpoints. An example based on a recently conducted phase II sarcoma trial illustrates the method. Copyright (C) 2012 John Wiley \& Sons, Ltd.
\end{abstract}

Keywords: Bayesian; decision theory; time to event; phase II clinical trial; two-stage design

\section{Introduction}

In phase II cancer clinical trials, the standard approach consists of a single-arm design where a single binary endpoint is compared with a specified target value. The sample sizes are typically small, maybe 30 70 patients. Improvements to the study could be made by increasing the sample size, using randomization and using an endpoint that is more informative than a binary one. Limitations on the available number of patients frequently limits power for a randomized study. Our focus in this paper will be on enhancing the trials using nonbinary endpoints. We will consider designs with failure-time endpoints, measuring time until some event, such as a device-related complication, disease progression, relapse, or death. Progression-free survival (PFS) is increasingly used as an endpoint for cancer clinical trials, and a recent review suggests that PFS is being utilized more commonly and may predict for greater success in the phase III setting [1]. Using PFS as an endpoint in single-arm studies does raise some issues concerning the possibility of bias in the comparison with the historical control group [2-4]. Although there is always the possibility of differences in the populations and in methods for assessing the endpoint between the trial and the control group, when using PFS, a consistent surveillance strategy for assessing progression is also needed.

A single-arm phase II trial is typically designed to accrue patients in two stages [5-7], with the Simon design being very popular. It will stop at stage I if a preset level of futility has been demonstrated, thereby reducing the number of patients exposed to an ineffective therapy. Similar to classical phase II studies, a study based on a time-to-event endpoint (such as median PFS or PFS rate at a specific clinical landmark point, $t_{0}$ ) will be deemed a success if there is sufficient statistical evidence to conclude that the endpoint

${ }^{a}$ Biostatistics Unit, University of Michigan Comprehensive Cancer Center, Ann Arbor, MI 48109, U.S.A.

${ }^{b}$ Department of Biostatistics, University of Michigan, Ann Arbor, MI 48109, U.S.A.

${ }^{c}$ Department of Internal Medicine, University of Michigan, Ann Arbor, MI 48109, U.S.A.

*Correspondence to: Lili Zhao, Biostatistics Unit, University of Michigan Comprehensive Cancer Center, Ann Arbor, MI 48109, U.S.A.

†E-mail:zhaolili@umich.edu 
exceeds, at a clinically relevant level, that of a relevant historical control. Many statistical designs are based on the probability that the patient survives to specific time $t_{0}$ without suffering the event. The most severe problem created by this approach is that a patient has to be followed-up for $t_{0}$ time to ensure that event has not occurred, and waiting until all patients in stage I complete the follow-up of $t_{0}$ may cause long recruitment suspension especially when $t_{0}$ is large. The impact of study suspension on accrual momentum and timeliness of the studies completion is often negative. A number of authors have formulated underlying statistical models and interim decision rules directly in terms of time-to-event variables to overcome this problem. Herndon [8] proposed a frequentist ad hoc approach to conducting two-stage phase II studies to avoid study suspension. Case and Morgan [9] proposed frequentist two-stage phase II designs using the estimator developed by Lin et al. [10] to minimize the expected sample size or expected total study length (ETSL) under $\mathrm{H}_{0}$. Huang et al. [11] modified their approach to protect type I error rate and improve robustness of the design. These designs $[9,11]$ are essentially an extension of the Simon designs with failure time endpoints using nonparametric statistics.

Researchers developed several Bayesian approaches to continuously monitor survival endpoints. Follman and Albert [12] used a Dirichlet process prior for the probabilities of the event on a large set of potential discretized event times. They compute an approximate posterior distribution that is a mixture of Dirichlet processes by using a data augmentation algorithm. Rosner [13] took a similar approach but used Gibbs sampling to generate posteriors. Cheung and Thall [14] constructed futility monitoring rules on the basis of an approximate posterior through a weighted average of beta distributions for one or more event times in phase II trials. These approaches incorporate the censored data into the posterior estimation in a nonparametric fashion. Thall et al. [15] developed model-based approaches to monitor time-to-event endpoints, assuming exponentially distributed failure times with an inverse gamma prior on the mean. They also examined the robustness of the method by assuming that the survival data follows a generalized gamma distribution. In the aforementioned Bayesian designs (e.g., $[14,15])$, decisions are made using the posterior distribution of the clinically relevant survival endpoints, and the futility monitoring rule is typically based on $P\left(p_{E}>p_{S}+\delta \mid\right.$ data $)<p_{L}$. Thus, the trial is stopped early if the posterior probability that $p_{E}$ (such as median survival or survival rate at a specific time point of the experimental treatment) exceeds a clinical meaningful threshold $(\delta)$ over the traditional treatment $p_{S}$ by less than a prespecified cutoff, $p_{L}$. The $p_{L}$ can be calibrated by simulations to obtain good trial properties such as probability of early termination, type I error, and power [16].

The property of Bayesian procedures to accumulate evidence based on updated data is very attractive in clinical trial designs. However, at the end of stage I, most investigators are interested in knowing what is the probability that the study would yield a significant result in favor of the new treatment if the study were to be continued to stage II, given what has been observed to date. Lachin [17] reviewed many frequentist approaches that construct stopping rules on the basis of an assessment of this idea using (predictive) conditional power. Pepe and Anderson [18] presented expressions for the types I and II error probabilities for use with time-to-event endpoints assuming current trends continue for survival data. Berry [19] is also a strong advocate for the use of predictive probabilities in making decisions. However, the aforementioned approaches (frequentist or Bayesian) use only the predictive distribution to make interim decisions. In this paper, we will weight the evidence for stopping or continuing on the basis of posterior and predictive distributions. Once the trial is stopped, the posterior distribution is used for making terminal decisions.

In the literature, researchers have developed decision-based Bayesian methods for binary endpoints [20-24]. Zhao and Woodworth [25] proposed a decision-based Bayesian approach for continually monitoring survival endpoints in single-arm phase II trials with medical devices, but the time-to-event data was assumed to be exponentially distributed.

In practice, the decision theoretic clinical trial designs remain relatively uncommon. The main reasons for this lack of application are fundamental concerns with the decision theoretic setup and practical difficulty of specifying a good loss function [26]. In decision-theoretic approaches, costs need to be specified, and in most situations, it is very hard to relate the costs to tangible quantities. Our strategy to make this approach feasible is to treat the cost parameters, defined in a simple loss structure, as tuning parameters, which are calibrated to achieve desired operating characteristics such as type I error and power. This approach alleviate concerns about difficulties in specifying the loss function, and as we will show, the properties of the decision theoretic designs appear to be very attractive.

Section 2 presents the general framework and methodology. Section 3 contains simulation studies to evaluate the properties of the proposed methods and compares the results to two frequentist designs. Section 4 contains results from a phase II sarcoma trial. Section 5 is the concluding discussion. 


\section{Statistics}

\section{Method}

The primary endpoint is a time-to-event outcome at some clinically meaningful landmark point $t_{0}$, such as 6 months or 1 year from the start of the treatment. Let $S\left(t_{0}\right)$ be the survival rate at $t_{0}$. Similar to most other designs with a binary endpoint of tumor response, decisions are made based on two hypotheses $\mathrm{H}_{0}$ : $S\left(t_{0}\right) \leqslant p_{1}$ that the true survival rate at $t_{0}$ is less than some uninteresting level $p_{1}$ and $\mathrm{H}_{1}: S\left(t_{0}\right) \geqslant p_{2}$ that the survival rate is at least some desirable target level $p_{2}$.

With right-censored data, the likelihood for $n$ subjects is

$$
L(\Theta \mid D)=\prod_{i=1}^{n}\left\{f\left(t_{i}\right)\right\}^{\delta_{i}}\left\{S\left(t_{i}\right)\right\}^{1-\delta_{i}}
$$

and

$$
S\left(t_{0}\right)=e^{-H\left(t_{0}\right)}, \quad \text { where } H\left(t_{0}\right)=\int_{0}^{t_{0}} h(u) \mathrm{d} u,
$$

where $\Theta$ are the parameters that determine the distribution $f(t)$ and $\mathrm{D}$ is the observed data, which include survival times, $t=\left(t_{1}, t_{2}, \ldots, t_{n}\right)$ and corresponding indicators of censoring, $\delta=\left(\delta_{1}, \delta_{2}, \ldots, \delta_{n}\right)$.

In this paper, we have chosen a very simple loss function as specified in Table I. There are two possible wrong decisions: (1) false rejection (type I error) and (2) false acceptance (type II error). $c_{2}$ is the penalty you are willing to pay for a false rejection decision over a false acceptance decision. In decision theory, $c_{2}$ could be a function of the sample size or could differ from stage I to stage II. In this paper, not to overcomplicate things, we consider $c_{2}$ as fixed and treat $c_{2}$ as a tuning parameter to control type I error and power. A rejection decision will be made if the posterior expected loss of making a rejection decision (e.g., $c_{2} P\left(S\left(t_{0}\right)<=p_{1} \mid D\right)$ ) is less than that of making an acceptance decision (e.g., $\left.P\left(S\left(t_{0}\right)>=p_{2} \mid D\right)\right)$, and an acceptance decision will be made otherwise. The higher the $c_{2}$, the less likely we are going to reject $\mathrm{H}_{0}$ to avoid the high penalty of a false rejection decision. But before we decide to stop and make a terminal decision (reject or accept $\mathrm{H}_{0}$ ), we should compare the risk of stopping at stage I to the 'expected' risk if the trial will be continued to stage II. The (posterior) Bayes risk of immediate stopping at stage I, denoted by $\rho_{0}\left(\pi^{1}\right)$, is defined as the minimum of the (posterior) expected losses under two decisions,

$$
\rho_{0}\left(\pi^{1}\right)=\min \left\{P\left(S\left(t_{0}\right) \geqslant p_{2} \mid D_{1}\right), c_{2} P\left(S\left(t_{0}\right) \leqslant p_{1} \mid D_{1}\right)\right\}
$$

where $D_{1}$ are the observed data up to stage I; $\pi^{1}$ is the posterior distribution of $S\left(t_{0}\right)$ given the observed data up to stage I, denoted by $f\left(S\left(t_{0}\right) \mid D_{1}\right)$.

The expected (posterior) Bayes risk of continuing to stage II, denoted by $\rho_{c}\left(\pi^{1}\right)$, is defined as,

$$
\begin{aligned}
\rho_{c}\left(\pi^{1}\right) & =E_{D_{2} \mid D_{1}}\left[\rho_{0}\left(\pi^{2}\right)\right]+c_{3} \\
& =E_{D_{2} \mid D_{1}}\left[\min \left\{P\left(S\left(t_{0}\right) \geqslant p_{2} \mid D_{2}\right), c_{2} P\left(S\left(t_{0}\right) \leqslant p_{1} \mid D_{2}\right)\right\}\right]+c_{3}
\end{aligned}
$$

\begin{tabular}{|c|c|c|c|c|}
\hline & & \multicolumn{3}{|c|}{ True status of the treatment } \\
\hline & & & Noninfe & \\
\hline & & Inferior & Neither & Superior \\
\hline & & $\left(S\left(t_{0}\right) \leqslant p_{1}\right)$ & $\left(p_{1}<S\left(t_{0}\right)<p_{2}\right)$ & $\left(S\left(t_{0}\right) \geqslant p_{2}\right)$ \\
\hline \multirow{2}{*}{ Decision } & Accept $\mathrm{H}_{0}$ & 0 & 0 & 1 \\
\hline & Reject $\mathrm{H}_{0}$ & $c_{2}$ & 0 & 0 \\
\hline
\end{tabular}


where $\pi^{2}$ is the posterior distribution of $S\left(t_{0}\right)$ given the observed data up to stage II, denoted by $f\left(S\left(t_{0}\right) \mid D_{2}\right), c_{3}$ is the cost of running the trial to the final stage, which could be a function of the sample size or study length in stage II. In this paper, $c_{3}$ is also fixed to control the probability of stopping at the end of stage I. The higher the $c_{3}$, the more likely the trial will be halted at stage I to avoid the high cost of continuing the trial. $E_{D_{2} \mid D_{1}}\left[\rho_{0}\left(\pi^{2}\right)\right]$ defined in (1) can be approximated by

$$
E_{D_{2} \mid D_{1}}\left[\rho_{0}\left(\pi^{2}\right)\right] \approx \frac{1}{B} \sum_{l=1}^{B}\left[\rho_{0}\left(\pi^{2, l}\right)\right] \quad \text { when } B \text { is large. }
$$

where $\pi^{2, l}$ is $f\left(S\left(t_{0}\right) \mid D_{2, l}\right)$ and $D_{2, l}(l=1,2, \ldots, B)$ are the random samples of potential datasets $D_{2}$ generated given $D_{1}$.

In brief, the decision rule is as follows:

- At the end of stage I

- if $\rho_{0}\left(\pi^{1}\right) \leqslant \rho_{c}\left(\pi^{1}\right)$, then stop and

$*$ Reject $\mathrm{H}_{0}$ if $\frac{P\left(S\left(t_{0}\right)>p_{2} \mid D_{1}\right)}{P\left(S\left(t_{0}\right)<p_{1} \mid D_{1}\right)}>c_{2}$

* Accept otherwise

- else if $\rho_{0}\left(\pi^{1}\right)>\rho_{c}\left(\pi^{1}\right)$, then continue to stage II

- At the end of stage II, stop and

- Reject $\mathrm{H}_{0}$ if $\frac{P\left(S\left(t_{0}\right)>p_{2} \mid D_{2}\right)}{P\left(S\left(t_{0}\right)<p_{1} \mid D_{2}\right)}>c_{2}$

- Accept otherwise

The aforementioned decision rules are attractive for two reasons. First, the terminal decision rule is constructed using the ratio of two posterior probabilities under two hypotheses (e.g., similar to posterior odds). Second, the decision to 'stop' or 'continue' is the result of weighting the evidence between the observed data and the data that will be observed if the trial would continue.

Once the posterior distribution of $\Theta$ is known, the distribution of $S\left(t_{0}\right)$, as a function of $\Theta$, can be computed using Monte Carlo methods. Therefore, it is easy to calculate the posterior probabilities under the two hypotheses. It is, however, much harder to calculate the Bayes risk of continuation, which involves the posterior predictive distribution of $D_{2}$ given $D_{1}$ in the presence of censoring. In the following section, we derive algorithms to estimate $E_{D_{2} \mid D_{1}}\left[\rho_{0}\left(\pi^{2}\right)\right]$ for exponential failure time distributions, Weibull distributions, and time-to-event data that do not follow any parametric distribution.

\subsection{Method assuming exponential distribution}

Assume the time-to-event data follows an exponential distribution, $f(t)=\lambda e^{-\lambda t}$ for $t>0$, and the primary endpoint $S\left(t_{0}\right)$ is defined as $S\left(t_{0}\right)=e^{-\lambda t_{0}}$.

The likelihood function for $\lambda$ at stage $k$ is $L\left(\lambda \mid D_{k}\right)=\lambda^{f^{k}} e^{-\lambda e^{k}}$, where $f^{k}$ is the total number of failures and $e^{k}$ is the total exposure time up to stage $k(k=1,2)$. These are sufficient statistics to estimate $\lambda$. $D$ then can be simplified to $D_{1}=\left(f^{1}, e^{1}\right)$ and $D_{2}=\left(f^{2}, e^{2}\right)$. For mathematical convenience, we use the conjugate prior for $\lambda$ such that $\lambda \sim \Gamma\left(\alpha_{0}, \beta_{0}\right)$. Then, the posterior distribution is easily determined to be a Gamma distribution, $f\left(\lambda \mid D_{k}\right) \sim \Gamma\left(\alpha_{0}+f^{k}, \beta_{0}+e^{k}\right)$.

Given the data up to stage I $\left(D_{1}\right)$, we can simulate the patients' survival data in stage II. The number of patients in stage II (denoted by $N_{2}$ ) has two parts: (1) the number of event-free patients that have not reached the final study point at the end of stage I; and (2) the number of new patients recruited in stage II. Because of the "lack of memory" property of the exponential distribution, those patients that are event free at the end of stage I are conditionally exchangeable with patients who are recruited in stage II, and their survival times in stage II will also be distributed as an exponential distribution with rate $\lambda \sim \Gamma\left(\alpha_{0}+f^{1}, \beta_{0}+e^{1}\right)$. Zhao and Woodworth [25] defined the algorithm to obtain samples of $D_{2}$ given $D_{1}$.

\subsection{Method assuming Weibull distribution}

The Weibull distribution, denoted by $\mathcal{W}(\alpha, \gamma)$, is $f(t)=\alpha \gamma t^{\alpha-1} e^{-\gamma t^{\alpha}}$ for $t>0, \alpha>0$, and $\gamma>0$. Let $\Theta=(\alpha, \gamma)$ and the primary endpoint is $S\left(t_{0}\right)=e^{-\gamma t_{0}^{\alpha}}$.

In this case, $D_{k}=\left(t^{k}, \delta^{k}\right), k=1,2 . t^{k}$ includes all survival times for patients enrolled up to stage $k$, and $\delta^{k}$ are the corresponding censoring indicators. Unlike the exponential distribution, there are no 
simple sufficient statistics and no simple posterior distributions for $\alpha$ and $\gamma$. MCMC methods will be used to estimate these two parameters.

This Weibull model can be expressed as a $\log$-linear model as $\log t_{i}=\mu+\sigma \epsilon_{i}$ [27], where $\mu=$ $-\log (\gamma) / \alpha$ and $\sigma=1 / \alpha$. The density of the $\log$ time, $y_{i}=\log t_{i}$, is given by

$$
f\left(y_{i}\right)=\frac{1}{\sigma} \exp \left(z_{i}-e^{z_{i}}\right), \text { where } z_{i}=\frac{y_{i}-\mu}{\sigma}
$$

If we assign $\mu$ a uniform prior and for $\sigma$ the usual noninformative prior proportional to $1 / \sigma$, then the posterior density is given by

$$
f\left(\mu, \sigma \mid D_{k}\right) \propto \frac{1}{\sigma} L\left(\mu, \sigma \mid D_{k}\right), \quad k=1,2
$$

Metropolis-Hasting algorithms can easily be applied to estimate $\mu$ and $\sigma$.

Given the data up to stage I $\left(D_{1}=\left(t^{1}, \delta^{1}\right)\right)$, survival times for patients enrolled in stage II will be generated from a Weibull distribution with the updated $\Theta$ from stage I. For any patient $i$ that is censored at the end of stage I and has not reached the final study point, the probability that the patient will survive additional time $x_{i}$ is expressed as

$$
S\left(x_{i}\right)=P\left(t>t_{i}^{1}+x_{i} \mid t>t_{i}^{1}\right)=e^{-\gamma\left(\left(t_{i}^{1}+x_{i}\right)^{\alpha}-t_{i}^{1 \alpha}\right)}
$$

To simulate this additional time, we simulate $S\left(x_{i, l}\right)$ from $U(0,1)$, and solving for $x$ gives

$$
x_{i, l}=\left\{\frac{-\log \left(S\left(x_{i, l}\right)\right)}{\gamma_{l}}+t_{i}^{1{ }^{\alpha_{l}}}\right\}^{\frac{1}{\alpha_{l}}}-t_{i}^{1},
$$

where $\alpha_{l}$ and $\gamma_{l}$ are from the $l$ th MCMC iteration. Then, $x_{i, l}, \quad l=1, \ldots, B$ are random samples from the posterior predictive distribution of the remaining survival time of patient $i$ in stage II.

Let $t_{i, l}^{2}=\min \left\{t_{i}^{1}+x_{i, l}, M_{i}\right\}, \delta_{i, l}^{2}=1$ if $t_{i, l}^{2}<M_{i}$ and $\delta_{i, l}^{2}=0$ otherwise, where $M_{i}$ is the maximum follow-up for patient $i$ defined from the enrollment to the final study time point. We repeat this process for all patients $\left(i=1, \ldots, N_{2}\right)$ in stage II, and together with the observed survival data of patients that had event, we obtain $D_{2, l}$. Then, $D_{2,1}, \ldots, D_{2, B}$ form a random sample from the posterior predictive distribution of $f\left(D_{2} \mid D_{1}\right)$.

Similar methods can be developed assuming Gamma or log-normal distributions.

\subsection{Grouped-data method}

In practice, interval-censored survival data is common in medical settings where the patient's disease status is evaluated periodically by tests such as magnetic resonance imaging or computed axial tomography scan. The actual time of any patients disease progression is not available, rather, it is only known whether progression occurred during each time interval between successive examinations. To account for this type of interval censoring, we propose a nonparametric method in this section, which handles the interval-censored data for various time-to-event data distributions.

Given the data up to stage I $\left(D_{1}\right)$, we construct a finite partition of the time axis, $0<s_{1}<s_{2}<$ $\cdots<s_{J}$, with $s_{J}=t_{0}$. Thus, we have the $J$ disjoint intervals $\left(0, s_{1}\right],\left(s_{1}, s_{2}\right], \ldots,\left(s_{J-1}, s_{J}\right]$, and $I_{j}=\left(s_{j-1}, s_{j}\right]$. The value of $s_{j}(j=1, \ldots, J)$, in the interval-censored case, should be determined by the intended gap between two consecutive scheduled appointments. For example, the scheduled appointment is every 2 months for the first half year, then the intervals can be set up as $s_{1}=2, s_{2}=4, s_{3}=6$, and $J=3$. For studies where the evaluation is more frequent, the value of $J$ would be much larger.

The observed data $D$ is assumed to be available as grouped within these intervals such that $D_{k}=$ $\left(\mathcal{R}_{j}^{k}, \mathcal{D}_{j}^{k}: j=1,2, \ldots, J\right)$, where $\mathcal{R}_{j}^{k}$ is the risk set and $\mathcal{D}_{j}^{k}$ is the failure set of the $j$ th interval $I_{j}$ up to stage $k$. Let $h_{j}$ denote the increment in the cumulative baseline hazard in the $j$ th interval, that is,

$$
h_{j}=H_{0}\left(s_{j}\right)-H_{0}\left(s_{j-1}\right), \quad j=1,2, \ldots, J
$$


and

$$
S\left(t_{0}\right)=\exp \left(-\sum_{j=1}^{J} h_{j}\right)
$$

The grouped data likelihood is

$$
L\left(\mathbf{h} \mid D_{k}\right) \propto \prod_{j=1}^{J} G_{j}^{k}, \quad \mathbf{h}=\left(h_{1}, h_{2}, \ldots, h_{J}\right)
$$

and

$$
G_{j}^{k}=\exp \left\{-h_{j}^{k}\left(r_{j}^{k}-d_{j}^{k}\right)\right\}\left\{1-\exp \left(-h_{j}^{k}\right)\right\}^{d_{j}^{k}}
$$

where $r_{j}^{k}$ and $d_{j}^{k}$ are the number of subjects in the sets $\mathcal{R}_{i}^{k}$ and $\mathcal{D}_{j}^{k}$ up to stage $k$, respectively.

The Gamma process is used as a prior for the cumulative baseline hazard function $H_{0}(t)$ [28] such that

$$
H_{0} \sim \mathcal{G P}\left(\tau_{0} H^{*}, \tau_{0}\right),
$$

where $H^{*}(t)$ is an increasing function with $H^{*}(0)=0 . H^{*}$ is assumed to be a Weibull distribution with hyperparameter $\eta_{0}$ and $\kappa_{0}$, such that $H^{*}(t)=\eta_{0} t^{\kappa_{0}}$. $\tau_{0}$ is a positive scalar quantifying the degree of prior confidence in $H^{*}(t)$. If $\kappa_{0}=1$, this simplifies as an exponential distribution with rate $\eta_{0}$.

The Gamma process prior in (8) implies that $h_{j}$ 's are independent and

$$
h_{j} \sim \Gamma\left(\tau_{0}\left(H^{*}\left(s_{j}\right)-H^{*}\left(s_{j-1}\right)\right), \tau_{0}\right) .
$$

With the likelihood and priors set up as that previously discussed, we can write the posterior distribution of $\mathbf{h}$ as specified in [29]

$$
f\left(\mathbf{h} \mid D_{k}\right) \propto \prod_{j=1}^{J} G_{j}^{k} h_{j}^{\tau_{0}\left(H^{*}\left(s_{j}\right)-H^{*}\left(s_{j-1}\right)\right)-1} e^{\tau_{0} h_{j}}
$$

We can carry out the following Gibbs sampling scheme sampling $h_{j}$ from

$$
\pi\left(h_{j} \mid \mathbf{h}^{-j}, D_{k}\right) \propto G_{j}^{k} h_{j}^{\tau_{0}\left(H^{*}\left(s_{j}\right)-H^{*}\left(s_{j-1}\right)\right)-1} e^{\tau_{0} h_{j}}
$$

where $\mathbf{h}^{-j}$ denote the $\mathbf{h}$ vector without $j$ th component. Plugging in the form for $G_{j}^{k}$ from Equation (7), we have

$$
\pi\left(h_{j} \mid \mathbf{h}^{-j}, D_{k}\right) \propto h_{j}^{\tau_{0}\left(H^{*}\left(s_{j}\right)-H^{*}\left(s_{j-1}\right)\right)-1}\left(1-e^{-h_{j}}\right)^{d_{j}^{k}} e^{-\left(\tau_{0}+r_{j}^{k}-d_{j}^{k}\right) h_{j}} .
$$

Survival times are approximated by piecewise constant hazard survival model. Thus, the memoryless property of the exponential distribution holds in each interval $I_{j}, j=1,2, \ldots, J$. Given data observed up to stage I $\left(D_{1}\right)$, survival times for patients in stage II $\left(i=1, \ldots, N_{2}\right)$ will be generated on the basis of the updated $h_{1}, \ldots, h_{J}$. For any patient $i$ that is censored in interval $I_{j}$ at the end of stage I and has not reached the final study point, the remaining survival time, $x_{i, j, l}$, will first be generated from an exponential distribution with rate $\frac{h_{j}}{s_{j}-s_{j-1}}$, then moving from left to right along the time line until the event occurs or the final study time point is reached, which will result in generated survival times, $x_{i, j, l}, x_{i, j+1, l}, \ldots$ in intervals $I_{j}, I_{j+1}, \ldots$, respectively. Let $t_{i, j, l}=\min \left\{x_{i, j, l},\left(\min \left\{s_{j}, M_{i}\right\}-t_{i}^{1}\right)\right\}$; if $t_{i, j, l}=s_{j}-t_{i}^{1}$ (no event occurred in $I_{j}$ and the final study point has not been reached), we will move to $I_{j+1}$. If no event occurred in $I_{j+1}$ and $M_{i} \geqslant s_{j+1}$, then $t_{i, j+1, l}=s_{j+1}-s_{j}$. Finally, 


\section{Statistics}

$t_{i, l}^{2}=t_{i}^{1}+t_{i, j, l}+t_{i, j+1, l}+\ldots$ For patients enrolled in stage II, this process starts at $I_{1}$. We repeat this process for all patients $\left(i=1, \ldots, N_{2}\right)$ in stage II to obtain a random sample from the posterior predictive distribution of $f\left(D_{2} \mid D_{1}\right)$.

We note that with the use of a large number of intervals, this grouped-data method can be viewed as a nonparametric method for general distributions that would be applicable even if the data were not interval censored.

\section{Simulation studies}

In this section, we ran simulations to investigate properties of the proposed designs (Bayes designs) including type I error, power, probability of early stopping (PET), expected sample size, and ETSL under both null and alternative hypotheses. In the simulation setup, we assumed the landmark time point, $t_{0}$, to be 6 months; we took the interim analysis 1 day before the first patient of stage II was enrolled such that there is no trial suspension and defined the final study point as 6 months after the enrollment of the last patient; the maximum follow-up per patient is 12 months; we sampled the patient arrival time from a Poisson distribution with a rate of 0.1 so that one patient arrives an average of every 10 days.

In Simon's designs, we dichotomize the survival time into a binary variable $I\left(t \leqslant t_{0}\right)$. With such designs, the trial will continue immediately to stage II once the number of successes reaches the threshold rather than waiting for all enrolled patients to complete the follow-up and stops immediately if there is no hope that the threshold will be met. For example, consider the situation where three successes of 10 is needed for the trial to continue to stage II. If only one success is observed for the first nine patients that had completed the follow-up, the trial should stop even though the last patient has not completed the follow-up because we would observe at most two successes in this case. For the Simon designs, we will consider both the MiniMax and the optimal designs. To be comparable with the Simon's designs, which only allow early stopping for futility, the stopping rule in the Bayes design is modified as

$$
\rho_{c} \geqslant \rho_{0} \text { and } \frac{P\left(S\left(t_{0}\right)>p_{2} \mid D_{1}\right)}{P\left(S\left(t_{0}\right)<p_{1} \mid D_{1}\right)} \leqslant c_{2}
$$

This 'Stop only for futility' rule is used throughout the simulations unless specified otherwise.

As well as comparing with the Simon designs, we will also make comparisons with the method described in [11]. This method prevents trial suspension by using Nelson-Aalen estimates of the survival calculated at different calendar times to account for the information available from those with partial follow-up. We implemented this method using the R program OptimPhase2.

\subsection{Effect of the cost parameters}

In this simple loss structure, cost parameter $c_{2}$ controls the trade-off between types I and II errors and cost parameter $c_{3}$ controls the PET. In this section, we investigated the effect of $c_{2}$ and $c_{3}$ while fixing the other. We calculated total sample size $(n=47)$ and the sample size in stage I $\left(n_{1}=24\right)$ from the Simon MiniMax design by restricting type I error to be 0.05 and type II error to be 0.15 with the two decision thresholds $p_{1}=0.1$ and $p_{2}=0.25$. We generated survival data from an exponential distribution with rate $-\frac{\log S\left(t_{0}\right)}{t_{0}}$, where $S\left(t_{0}\right)=0.1$ or 0.25 . We used an uninformative Gamma prior distribution, with mean 0.0001 and rate 0.0001 , for $\lambda$.

Figure 1 demonstrates the effect of $c_{3}$ when $c_{2}$ is fixed to be 5. The highest $c_{3}$ (e.g., $c_{3}=0.1$ ) gives the highest PET across different $S\left(t_{0}\right)$, and $c_{3}$ of 0.03 provides similar PET as the MiniMax design. In Figure $2, c_{3}$ is fixed to be 0.03 . The highest $c_{2}\left(\right.$ e.g., $\left.c_{2}=10\right)$ has the lowest probability of rejecting the $\mathrm{H}_{0}$, resulting in the lowest power at $S\left(t_{0}\right)=0.25$ as well as the lowest type I error at $S\left(t_{0}\right)=0.1$. We found that the choice of $c_{2}=3$ and $c_{3}=0.03$ gives type I error of 0.03 and power of 0.9 . To obtain approximately type I error of 0.05 and power of approximately 0.85 , to match the MiniMax design, we reduced the sample size by $32 \%$ to $n=32$ given $c_{2}=3$ and $c_{3}=0.03$. With this sample size, we investigated the operating characteristics of trials with different design parameters such as different hypotheses ( $p_{1}$ and $p_{2}$ ), different patient accrual rates, different timing of the interim look, a stopping rule that allows stopping for both efficacy and futility, and different prespecified values for types I and II errors. Without loss of generality, we used the exponential method for data that are exponentially distributed. 


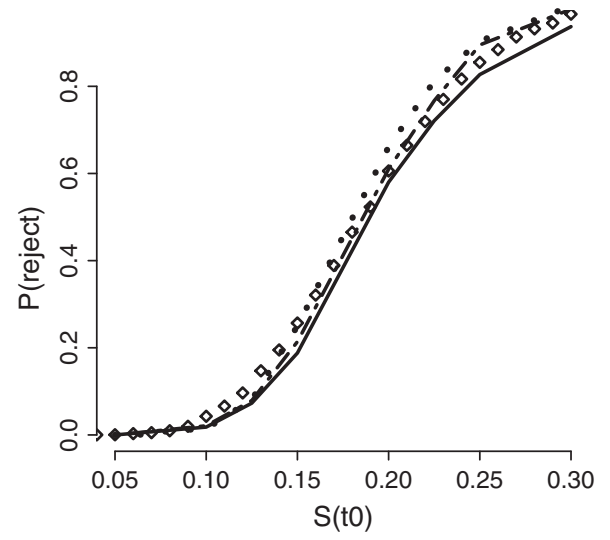

(a)

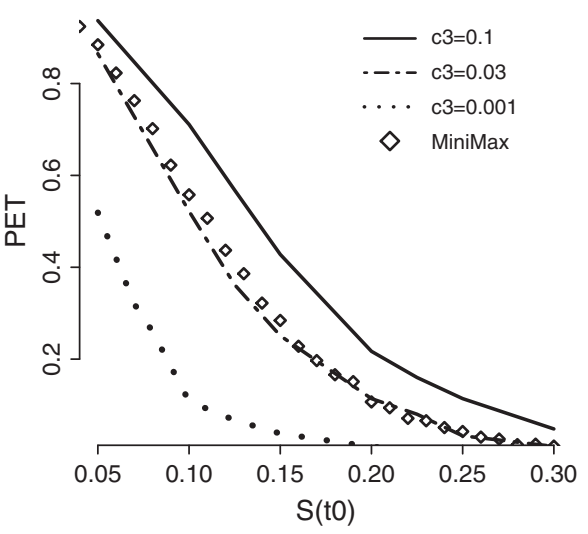

(b)

Figure 1. (a) Probability of rejecting $\mathrm{H}_{0}$ for various $S\left(t_{0}\right)$, given three choices of $c_{3}$ with $c_{2}$ fixed to be 5 , and (b) the corresponding probability of early stopping when $p_{1}$ is true for various $S\left(t_{0}\right)$, given three choices of $c_{3}$ with $c_{2}$ fixed to be 5 (on the basis of 1000 trial simulations and $1000(B=1000)$ simulations in calculating the expected Bayes risk of continuation).

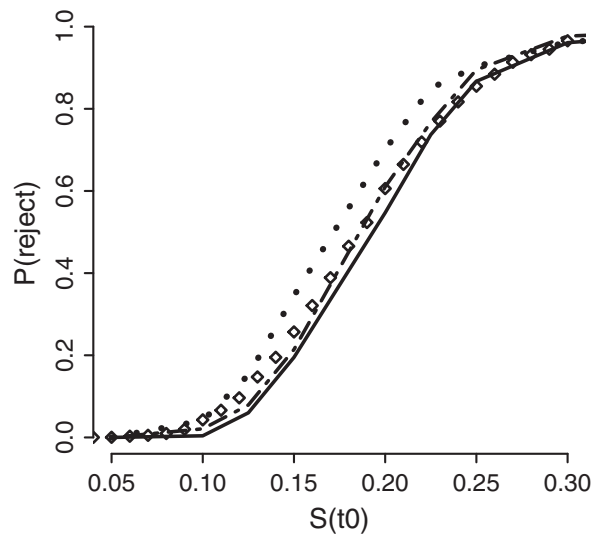

(a)

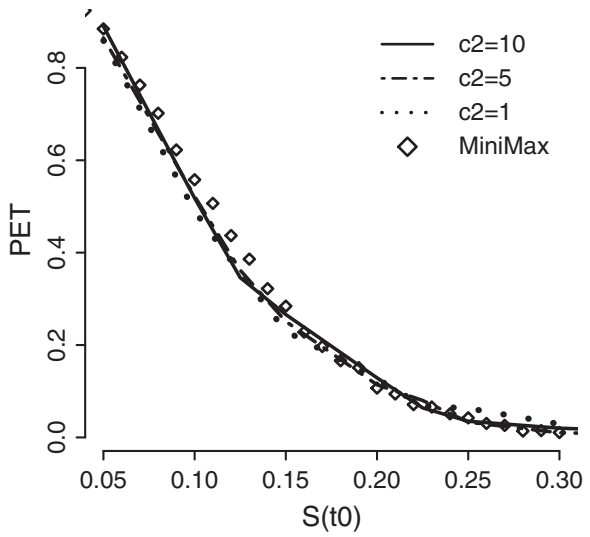

(b)

Figure 2. (a) Probability of rejecting $\mathrm{H}_{0}$ for various $S\left(t_{0}\right)$, given three choices of $c_{2}$ with $c_{3}$ fixed to be 0.03 , and (b) the corresponding probability of early stopping when $p_{1}$ is true for various $S\left(t_{0}\right)$, given three choices of $c_{2}$ with $c_{3}$ fixed to be 0.03 (on the basis of 1000 trial simulations and $1000(B=1000)$ simulations in calculating the expected Bayes risk of continuation).

\subsection{Trial properties with different design parameters}

3.2.1. Trials with different hypotheses. We base the results shown in Table II on 5000 simulations of the same trial and $1000(B=1000)$ simulations in calculating the Bayes risk of continuation. With $c_{2}=3$ and $c_{3}=0.03$, all the studied cases have type I error of approximately 0.05 and power of approximately 0.85 using about $30 \%$ less total sample size than the MiniMax design. The Bayes designs significantly shorten the average trial duration with much smaller expected sample sizes, compared with other designs. For example, in the first scenario, it shortens the total study length by 4 months if $S\left(t_{0}\right)$ is 0.1 and by 7 months if $S\left(t_{0}\right)$ is 0.25 , compared with the MiniMax design. The savings in sample size are even more under the alternative hypothesis because of the dramatically reduced total sample size and seamless enrollment. The PET is low in the Bayes design under the null hypothesis. This, nonetheless, does not result in longer trial duration and larger expected sample size because of the dramatically reduced total sample size. And increasing $n_{1}$ would increase the PET as will be presented in the next section. The outperformance of the Bayes designs over the Simon's designs is mainly due to making use of more information with the time-to-event data rather than the dichotomized data in Simon's designs. 


\begin{tabular}{|c|c|c|c|c|c|c|c|}
\hline \multirow[b]{2}{*}{ Design } & \multirow[b]{2}{*}{$n\left(n_{1}\right)^{\mathrm{a}}$} & \multicolumn{3}{|c|}{ Under $\mathrm{H}_{0}$} & \multicolumn{3}{|c|}{ Under $\mathrm{H}_{1}$} \\
\hline & & PET & EN & $\mathrm{ETSL}^{\mathrm{b}}$ & PET & EN & ETSL \\
\hline \multicolumn{8}{|c|}{$0.10 v s 0.25$} \\
\hline MiniMax & $47(24)$ & 0.57 & 34 & 17 & 0.04 & 46 & 23 \\
\hline Optimal $^{\mathrm{d}}$ & $50(20)$ & 0.68 & 30 & 15 & 0.09 & 47 & 24 \\
\hline $\mathrm{H}$-ETSL ${ }^{\mathrm{e}}$ & $52(33)$ & 0.62 & 40 & 16 & 0.09 & 50 & 22 \\
\hline Bayes & $32(16)$ & 0.34 & 27 & 13 & 0.04 & 31 & 16 \\
\hline \multicolumn{8}{|c|}{$0.2 v s 0.4$} \\
\hline MiniMax & $37(17)$ & 0.55 & 26 & 15 & 0.05 & 36 & 21 \\
\hline Optimal & $51(17)$ & 0.76 & 25 & 13 & 0.12 & 47 & 25 \\
\hline H-ETSL & $49(31)$ & 0.67 & 37 & 14 & 0.08 & 48 & 21 \\
\hline Bayes & $26(13)$ & 0.25 & 23 & 12 & 0.03 & 26 & 14 \\
\hline \multicolumn{8}{|c|}{$0.3 v s 0.45$} \\
\hline MiniMax & $76(35)$ & 0.51 & 55 & 24 & 0.04 & 75 & 33 \\
\hline Optimal & $86(29)$ & 0.64 & 49 & 22 & 0.09 & 81 & 36 \\
\hline H-ETSL & $98(48)$ & 0.68 & 64 & 23 & 0.08 & 94 & 37 \\
\hline Bayes & $54(27)$ & 0.38 & 44 & 18 & 0.04 & 53 & 23 \\
\hline \multicolumn{8}{|c|}{$0.5 v s 0.7$} \\
\hline MiniMax & $44(22)$ & 0.60 & 31 & 16 & 0.04 & 43 & 24 \\
\hline Optimal & $52(17)$ & 0.67 & 28 & 15 & 0.09 & 49 & 26 \\
\hline H-ETSL & $66(37)$ & 0.69 & 46 & 17 & 0.06 & 64 & 27 \\
\hline Bayes & $30(15)$ & 0.23 & 27 & 13 & 0.03 & 30 & 16 \\
\hline
\end{tabular}

PET, probability of early stopping; EN, expected sample size; ETSL, expected total study length.

${ }^{\mathrm{a}} n$ in the Bayes designs is about $70 \%$ of the sample size calculated from the corresponding MiniMax design, and $n_{1}$ is half of the total sample size.

${ }^{\mathrm{b}}$ The unit of ETSL is months.

${ }^{\mathrm{c}}$ MiniMax design is targeted at minimizing the maximum sample size under the null hypothesis.

${ }^{\mathrm{d}}$ Optimal design is targeted at minimizing the expected sample size under the null hypothesis.

${ }^{\text {e}} \mathrm{H}$-ETSL is targeted at minimizing the ETSL. The sample size is calculated using the OptimDes function in the OptimPhase 2 package in $\mathrm{R}$ while fixing the power 0.85 and type I error 0.05 . The operating characteristics are obtained with the SimDes function in the OptimPhase 2 package.

On the contrary, the H-ETSL design, although it successfully avoids trial suspension, will not necessarily reduce the trial duration.

The optimal designs give smaller expected sample size and shorter averaged trial duration than the MiniMax designs under the null hypotheses (the opposite is true under the alternatives). We will use the optimal design for the comparisons for the rest of the article.

3.2.2. Trials with the interim look taken at a different time point. As illustrated in Table III, larger $n_{1}$ gives higher PET under the null hypothesis. Increasing $n_{1}$ from 16 to 24 further reduces the trial duration by 1 month. This fact suggests that interim analysis may be performed after we accumulate more data information (larger $n_{1}$ or longer follow-up), which will provide us with more evidence to terminate the trial early if the null hypothesis is true, thereby increasing the PET and shortening the trial duration especially when the patient actual is fast. In contrast with the Bayes designs, smaller $n_{1}$ in Simon's designs gives larger PET when the optimal design is compared with the MiniMax design (Table II). This reflects a fundamental problem in frequentist designs based on hypothesis testing, in which the hypotheses are set up to collect evidence to prove the treatment is promising (i.e., reject the $\mathrm{H}_{0}$ ), which leads to the tendency to declare the treatment bad with smaller sample sizes. 


\begin{tabular}{|c|c|c|c|c|c|c|}
\hline \multirow[b]{2}{*}{$n\left(n_{1}\right)$} & \multicolumn{3}{|c|}{ Under $\mathrm{H}_{0}$} & \multicolumn{3}{|c|}{ Under $\mathrm{H}_{1}$} \\
\hline & PET & EN & ETSL & PET & EN & ETSL \\
\hline $32(8)$ & 0.12 & 29 & 15 & 0.03 & 31 & 16 \\
\hline $32(16)$ & 0.34 & 27 & 13 & 0.04 & 31 & 16 \\
\hline $32(24)$ & 0.61 & 27 & 12 & 0.06 & 31 & 16 \\
\hline
\end{tabular}

PET, probability of early stopping; EN, expected sample size; ETSL, expected total study length.

3.2.3. Trials with stopping for both efficacy and futility. Stopping for both futility and efficacy is naturally embedded within the decision-theoretic framework. As demonstrated in Table IV, type I error and power are increased slightly by allowing stopping for efficacy as well as for futility. Increasing $c_{2}$ (such as $c_{2}=4$ ) would lower the probability of rejection (that is, the type I error and power) by approximately $2 \%$.

3.2.4. Trials with different interpatient arrival times. As presented in Figure 3, the Bayes design has the smallest averaged trial duration for a wide range of accrual rates under both the null and alternative hypotheses. The H-ETSL design only shows some benefit over the optimal design under the alternative hypothesis when the accrual rate is very fast.

3.2.5. Trials with different type I error and power. Different clinical trials may have different requirements for the type I error and power. In cancer trials, other choices may be as follows: (1) type I error of $5 \%$ with power of $80 \%$ or (2) type I error of $10 \%$ with power of $90 \%$. With $70 \%$ of the total sample size calculated from the MiniMax design, we found, as shown in Table V, that $c_{2}=4$ and $c_{3}=0.03$ give type I error of approximately 0.05 and power of approximately 0.80 , and $c_{2}=1$ and $c_{3}=0.02$ give type I error of approximately 0.1 and power of approximately 0.90 .

\subsection{Simulation using the Weibull method}

Thus far, we have assumed that survival data is exponentially distributed. If the event rates are not constant, however, a more complex distribution is required. In this section, we applied the Weibull method for data generated from Weibull distributions with various shape parameters (denoted by $\alpha$ ) given $S\left(t_{0}\right)=0.1$ or 0.25 . We base the results in Table VI on 500 simulated trials and $250(B=250)$ simulations to calculate the expected Bayes risk of continuation. With $c_{2}=3$ and $c_{3}=0.03$, the type I error is approximately 0.05 and power is approximately 0.85 , regardless of different shape parameters.

\begin{tabular}{|c|c|c|c|c|c|c|c|c|}
\hline \multirow[b]{2}{*}{$n\left(n_{1}\right)$} & \multicolumn{4}{|c|}{ Under $\mathrm{H}_{0}$} & \multicolumn{4}{|c|}{ Under $\mathrm{H}_{1}$} \\
\hline & Type I error & PET & EN & ETSL & Power & PET & EN & ETSL \\
\hline $0.10 v s 0.25$ & & & & & & & & \\
\hline $\begin{array}{l}32(16) \\
0.2 v s 0.4\end{array}$ & 0.07 & 0.36 & 26 & 13 & 0.86 & 0.31 & 27 & 13 \\
\hline $\begin{array}{l}26(13) \\
\quad 0.3 v s 0.45\end{array}$ & 0.08 & 0.29 & 22 & 12 & 0.85 & 0.23 & 23 & 12 \\
\hline $\begin{array}{l}54(27) \\
\quad 0.5 v s .0 .7\end{array}$ & 0.07 & 0.39 & 43 & 18 & 0.85 & 0.31 & 46 & 19 \\
\hline $30(15)$ & 0.08 & 0.27 & 26 & 13 & 0.88 & 0.24 & 26 & 13 \\
\hline
\end{tabular}

PET, probability of early stopping; EN, expected sample size; ETSL, expected total study length. 


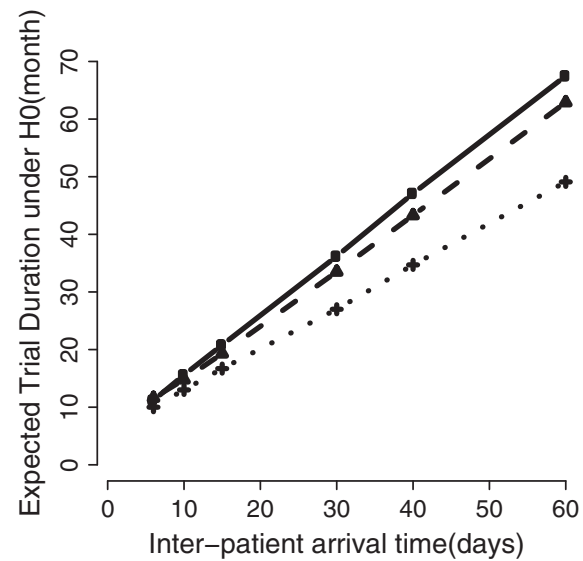

(a)

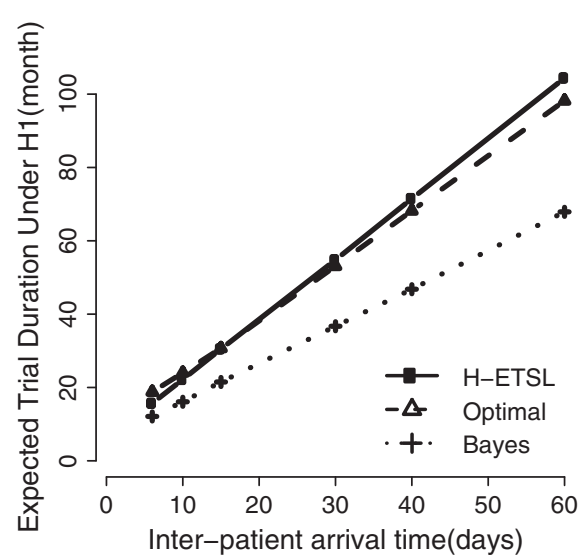

(b)

Figure 3. (a) Expected trial duration when $S\left(t_{0}\right)=0.1$ for various interpatient arrival times; and (b) expected trial duration when $S\left(t_{0}\right)=0.25$ for various interpatient arrival times.

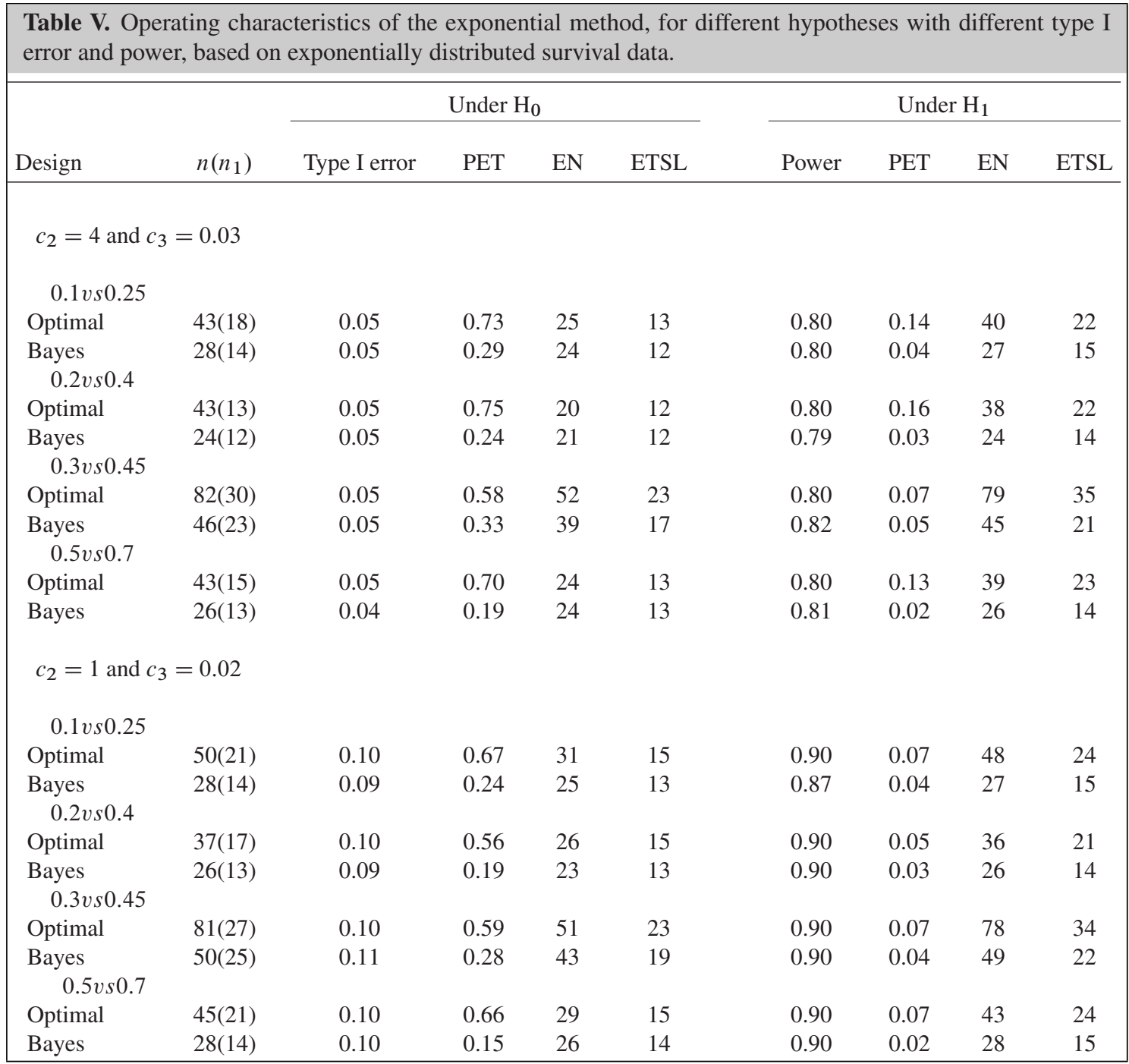

PET, probability of early stopping; EN, expected sample size; ETSL, expected total study length. 


\begin{tabular}{|c|c|c|c|c|c|c|c|}
\hline \multirow[b]{2}{*}{ Design } & \multirow[b]{2}{*}{$n\left(n_{1}\right)$} & \multicolumn{3}{|c|}{ Under $\mathrm{H}_{0}$} & \multicolumn{3}{|c|}{ Under $\mathrm{H}_{1}$} \\
\hline & & PET & EN & ETSL & PET & EN & ETSL \\
\hline & $\alpha=0.5$ & & & & & & \\
\hline Optimal & $50(20)$ & 0.68 & 30 & 14 & 0.09 & 47 & 24 \\
\hline H-ETSL & $53(31)$ & 0.66 & 39 & 15 & 0.09 & 51 & 22 \\
\hline Bayes & $32(16)$ & 0.36 & 26 & 13 & 0.04 & 31 & 16 \\
\hline Bayes & $32(24)$ & 0.58 & 27 & 12 & 0.03 & 32 & 16 \\
\hline \multirow[t]{2}{*}{ BayesII } & $32(16)$ & 0.38 & 26 & 12 & 0.34 & 27 & 13 \\
\hline & $\begin{array}{l}\alpha=1 \\
5\end{array}$ & 068 & 30 & 15 & 009 & 47 & 24 \\
\hline H-ETSL & $52(33)$ & $\begin{array}{l}0.00 \\
0.62\end{array}$ & 41 & $\begin{array}{l}15 \\
16\end{array}$ & $\begin{array}{l}0.09 \\
0.09\end{array}$ & $\begin{array}{l}47 \\
50\end{array}$ & $\begin{array}{l}24 \\
22\end{array}$ \\
\hline Bayes & $32(16)$ & 0.23 & 28 & 14 & 0.03 & 32 & 16 \\
\hline Bayes & $32(24)$ & 0.43 & 29 & 13 & 0.03 & 32 & 16 \\
\hline \multirow[t]{2}{*}{ BayesII } & $32(16)$ & 0.22 & 29 & 14 & 0.25 & 28 & 14 \\
\hline & $\alpha=1.5$ & & & & & & \\
\hline Optimal & $50(20)$ & 0.68 & 30 & 15 & 0.09 & 47 & 24 \\
\hline H-ETSL & $52(34)$ & 0.62 & 41 & 16 & 0.09 & 50 & 22 \\
\hline Bayes & $32(16)$ & 0.15 & 30 & 15 & 0.03 & 32 & 16 \\
\hline Bayes & $32(24)$ & 0.38 & 29 & 13 & 0.02 & 32 & 16 \\
\hline \multirow[t]{2}{*}{ BayesII } & $32(16)$ & 0.19 & 29 & 15 & 0.18 & 29 & 15 \\
\hline & $\alpha=2$ & & & & & & \\
\hline Optimal & $50(20)$ & 0.67 & 30 & 16 & 0.09 & 47 & 24 \\
\hline H-ETSL & $52(34)$ & 0.61 & 41 & 16 & 0.09 & 50 & 22 \\
\hline Bayes & $32(16)$ & 0.14 & 30 & 15 & 0.03 & 32 & 16 \\
\hline Bayes & $32(24)$ & 0.33 & 29 & 14 & 0.03 & 32 & 16 \\
\hline BayesII & $32(16)$ & 0.19 & 29 & 14 & 0.19 & 29 & 14 \\
\hline
\end{tabular}

PET, probability of early stopping; EN, expected sample size; ETSL, expected total study length.

Again, the Weibull method significantly shortens the trial duration with only $70 \%$ of the sample size from the MiniMax design. The PET decreases as the median failure time increases (as measured by $\alpha$ ) because more events (evidence) are observed with smaller median failure times, which leads to shorter trial duration and smaller expected sample size.

Table VI also suggests that waiting longer (larger $n_{1}$ ) to make the interim decision could result in higher PET and shorter trial duration under the null hypothesis (compare $n_{1}=16$ versus $n_{1}=24$ ).

3.3.1. Robustness with the Weibull method. We examined the robustness of the Weibull method for data generated from log-normal and Gamma distributions. As we can see from Table VII, the Weibull method is very robust in the cases studied.

\subsection{Simulation using the grouped-data method}

If time-to-event data can be assumed to follow a parametric distribution, the Weibull method could be performed because of its robustness presented in the last section. In this section, we evaluate a design for trials for which the majority of the events are observed at the scheduled tumor assessment time points. The grouped-data method was applied to survival data generated from various distributions such as Weibull, log-normal, and Gamma distributions. The time points of the grid were chosen to be $0<2<4<6$ under the assumption that the appointment is scheduled every 2 months. Prior parameters of $\tau_{0}=0.1$ and $\eta=0.014\left(\kappa_{0} \equiv 1\right)$ imply that a priori, $\mathrm{P}\left(S\left(t_{0}\right)<0.1\right)=0.16$ and $\mathrm{P}\left(S\left(t_{0}\right)>0.25\right)=0.8$. 


\begin{tabular}{|c|c|c|c|c|c|c|c|c|c|}
\hline \multirow[b]{2}{*}{ Design } & \multirow[b]{2}{*}{$n\left(n_{1}\right)$} & \multicolumn{4}{|c|}{ Under $\mathrm{H}_{0}$} & \multicolumn{4}{|c|}{ Under $\mathrm{H}_{1}$} \\
\hline & & Type I error & PET & EN & ETSL & Power & PET & EN & ETSL \\
\hline \multicolumn{10}{|c|}{$\mathcal{L} \mathcal{N}(\mu, 2)$} \\
\hline Optimal & $50(20)$ & 0.05 & 0.68 & 30 & 14 & 0.85 & 0.09 & 47 & 23 \\
\hline Bayes & $32(24)$ & 0.05 & 0.73 & 26 & 10 & 0.80 & 0.10 & 31 & 16 \\
\hline \multicolumn{10}{|c|}{$\mathcal{L} \mathcal{N}(\mu, 1)$} \\
\hline Optimal & $50(20)$ & 0.05 & 0.67 & 30 & 15 & 0.85 & 0.02 & 47 & 24 \\
\hline Bayes & $32(24)$ & 0.05 & 0.64 & 27 & 11 & 0.86 & 0.06 & 32 & 16 \\
\hline \multicolumn{10}{|c|}{$\mathcal{G}(1, b)$} \\
\hline Optimal & $50(20)$ & 0.05 & 0.68 & 30 & 15 & 0.85 & 0.09 & 47 & 24 \\
\hline Bayes & $32(24)$ & 0.05 & 0.41 & 29 & 13 & 0.85 & 0.04 & 32 & 16 \\
\hline \multicolumn{10}{|c|}{$\mathcal{G}(2, b)$} \\
\hline Optimal & $50(20)$ & 0.05 & 0.68 & 30 & 15 & 0.85 & 0.09 & 47 & 24 \\
\hline Bayes & $32(24)$ & 0.05 & 0.45 & 28 & 13 & 0.82 & 0.05 & 32 & 16 \\
\hline
\end{tabular}

PET, probability of early stopping; EN, expected sample size; ETSL, expected total study length.

But this prior distribution of $S\left(t_{0}\right)$ is flat, so that the influence of the prior on the posterior distribution is small. We increased $n_{1}$ from 24 to 30 to gain more information at the end of stage I.

With the results shown in Table VIII with 500 repeated trials and $250(B=250)$ simulations for the expected Bayes risk of continuation, the grouped-data method shortens the trial duration only under the alternative hypotheses because of the seamless recruitment. With the same total sample size as in the MiniMax design, the significantly lower PET in the Bayes design (even based on six more patients than in the MiniMax design) results in longer trial duration under the null hypothesis. With slower accrual or longer follow-up per patient, the trial could have higher PET. We also found that allowing stopping for both efficacy and futility increased the type I error to about 10\%-15\% (results not shown). Therefore, we would suggest that a trial only be allowed to stop for futility when the grouped-data method is applied.

\section{Application of the methods to a sarcoma clinical trial}

The primary objective of this trial is to evaluate the 6-month PFS rate in patients with advanced sarcoma treated with oral cyclophosphamide and sirolimus (OCR). The trial was originally designed using the Simon MiniMax design. Patients who are progression free and alive at 6 months following the initiation of study treatment will be considered a success. A $25 \%$ or greater 6-month PFS rate from OCR treatment would be considered as showing potential activity of the combination, thus worthy of further study. A $10 \%$ or less 6-month PFS rate from OCR is considered uninteresting for additional study. With a type I error rate of 5\%, the study of 47 evaluable patients will provide $85 \%$ power to detect an effective treatment. Tumor imaging will be performed every 2 months until tumor progression or until the patient completes 12 cycles of treatment. Each cycle is 28 days long. According to the MiniMax design, 24 patients will be enrolled in stage I. If three or more patients are alive and free from sarcoma progression 6 months after enrollment, an additional 23 patients will be enrolled in the second stage of the study. If nine or more of the 47 patients are alive and free of progression 6 months after enrollment, the proposed treatment will be declared as having anti-sarcoma activity worthy of further study. The trial is now closed for accrual, and the trial was paused for about 2 months until three patients reached 6 months follow-up and were free of progression and thereafter continued to stage II. The trial declared the OCR treatment as promising after nine patients were progression free after 6 months in stage II.

If we could design the trial using the Bayes design, we would choose $c_{2}=3$ and $c_{3}=0.03$ for type I error of 0.05 and power of 0.85 as specified in the MiniMax design. Then, we assume that patients 
Table VIII. Operating characteristics of the grouped-data method for hypothesis of 0.1 vs 0.25 , given type I error of approximately 0.05 and power of approximately 0.85 , based on Weibull, Gamma, and log-normal distributed survival data.

\begin{tabular}{|c|c|c|c|c|c|c|c|}
\hline \multirow[b]{2}{*}{ Design } & \multirow[b]{2}{*}{$n\left(n_{1}\right)$} & \multicolumn{3}{|c|}{ Under $\mathrm{H}_{0}$} & \multicolumn{3}{|c|}{ Under $\mathrm{H}_{1}$} \\
\hline & & PET & EN & ETSL & PET & EN & ETSL \\
\hline \multicolumn{8}{|c|}{$\mathcal{W}(0.5, \gamma)$} \\
\hline Optimal & $50(20)$ & 0.68 & 30 & 14 & 0.09 & 47 & 24 \\
\hline Bayes & $47(30)$ & 0.48 & 39 & 16 & 0.03 & 47 & 21 \\
\hline \multicolumn{8}{|c|}{$\mathcal{W}(1.5, \gamma)$} \\
\hline Optimal & $50(20)$ & 0.68 & 30 & 15 & 0.09 & 47 & 24 \\
\hline Bayes & $47(30)$ & 0.25 & 43 & 19 & 0.02 & 47 & 21 \\
\hline \multicolumn{8}{|c|}{$\mathcal{L} \mathcal{N}(\mu, 2)$} \\
\hline Optimal & $50(20)$ & 0.68 & 30 & 14 & 0.09 & 47 & 23 \\
\hline Bayes & $47(30)$ & 0.53 & 38 & 16 & 0.03 & 46 & 21 \\
\hline \multicolumn{8}{|c|}{$\mathcal{L} \mathcal{N}(\mu, 1)$} \\
\hline Optimal & $50(20)$ & 0.67 & 30 & 15 & 0.09 & 47 & 24 \\
\hline Bayes & $47(30)$ & 0.32 & 42 & 18 & 0.02 & 47 & 21 \\
\hline \multicolumn{8}{|c|}{$\mathcal{G}(1, b)$} \\
\hline Optimal & $50(20)$ & 0.68 & 30 & 15 & 0.09 & 47 & 24 \\
\hline Bayes & $47(30)$ & 0.33 & 41 & 18 & 0.03 & 47 & 21 \\
\hline \multicolumn{8}{|c|}{$\mathcal{G}(2, b)$} \\
\hline Optimal & $50(20)$ & 0.68 & 30 & 15 & 0.09 & 47 & 24 \\
\hline Bayes & $47(30)$ & 0.24 & 43 & 19 & 0.02 & 47 & 22 \\
\hline
\end{tabular}

PET, probability of early stopping; EN, expected sample size; ETSL, expected total study length.

\begin{tabular}{|c|c|}
\hline & Time to progression/death (days) \\
\hline Stage I $\left(D_{1}\right)$ & $\begin{array}{l}103,112,212^{+}, 210^{+}, 112,111,56,59,111,31 \\
142^{+}, 110,61,133^{+}, 133^{+}, 93^{+}, 51,87^{+}, 56 \\
74^{+}, 67^{+}, 53^{+}, 31^{+}, 11\end{array}$ \\
\hline Stage II $\left(D_{2}\right)$ & $\begin{array}{l}103,112,225,360^{+}, 112,111,56,59,111,31, \\
162,110,61,223,360^{+}, 225,51,253,56, \\
107,219,140,55,11,112,113,53,112,56, \\
184^{+}, 180^{+}, 56,57,54,61,28,56,56,49,100^{+}, 95, \\
85^{+}, 52,62,65^{+}, 56,42^{+}\end{array}$ \\
\hline
\end{tabular}

in stage II are enrolled 2 months earlier than their true enrollment dates such that there is no trial suspension at the end of stage I. The end of stage II is defined when nine patients reach the time $t_{0}$ and are free of progression under this hypothetical enrollment schedule. In contrast to the Simon's designs, which require waiting for one more event from the nine patients that are event free and have not been followed-up for 6 months, the Bayes design will make the interim decision on the basis of the data $D_{1}$ in Table IX.

In this trial, although the design should lead to interval censored data, the variation of patient scheduling, death without confirmed progression, and clinical progression that triggers an earlier detection of the progression, in our view, justify using a parametric distribution for the event time data. Because the mean estimate of $\alpha$ is about 1.6 based on the data $D_{1}$, the Weibull method was used. We then tried the Weibull method using $68 \%$ of the sample size as in the MiniMax design $(n=32)$ with $n_{1}=24$. In the first 


\section{Statistics}

Table X. Expected losses and decisions at two stages.

\begin{tabular}{|c|c|c|c|c|c|c|c|}
\hline \multirow[b]{2}{*}{$n\left(n_{1}\right)$} & \multirow[b]{2}{*}{ Action } & \multicolumn{4}{|c|}{ Stage I } & \multicolumn{2}{|c|}{ Stage II } \\
\hline & & ELoss & $\rho_{0}$ & $\rho_{c}$ & Decision & ELoss & Decision \\
\hline \multicolumn{8}{|l|}{ Weibull } \\
\hline \multirow[t]{2}{*}{$47(24)$} & Accept & 0.64 & 0.08 & 0.04 & Continue & 0.38 & Reject \\
\hline & Reject & 0.08 & & & & 0.02 & \\
\hline \multirow[t]{2}{*}{$32(24)$} & Accept & 0.64 & 0.08 & 0.05 & Continue & 0.75 & Reject \\
\hline & Reject & 0.08 & & & & 0.01 & \\
\hline \multicolumn{8}{|c|}{ Grouped data } \\
\hline \multirow[t]{2}{*}{$47(30)$} & Accept & 0.65 & 0.02 & 0.03 & Continue & 0.40 & Reject \\
\hline & Reject & 0.02 & & & & 0.02 & \\
\hline
\end{tabular}

design, the posterior expected loss of a rejection and an acceptance decision are 0.08 and 0.64 , respectively (see Table X). The smaller of these two is 0.08 , which is the Bayes risk of immediate stopping. Because it is larger than the expected Bayes risk of continuation $\left(\rho_{c}=0.04\right)$, the trial is continued to the second stage. At the end of the trial, the posterior expected loss of a rejection decision is smaller $(0.02$ vs 0.38 ). Therefore, we reject the $\mathrm{H}_{0}$. In the first two designs, $\rho_{0}$ is the same because $D_{1}$ is the same in both designs, whereas $\rho_{c}$ is smaller in the first design because more information (less risk) is expected if we would enroll more patients in the second stage given the same $c_{3}$.

We note that using the grouped-data method with the same time points of grids and priors as specified in the simulation studies, we would also reject $\mathrm{H}_{0}$.

All the three designs give the same conclusion as the MiniMax design that the OCR is effective, but the Bayes designs have shorter trial duration.

\section{Discussion}

There are three reasons for the use of decision-based Bayesian approach in designing clinical trials with interim looks. First, it is natural to make 'stop' or 'continue' decisions on the basis of the risk of 'stop' and 'continue' if the trial will continue to the final stage. Once we decide to stop, the terminal decision is based on the observed data alone (with noninformative priors). Second, the terminal decision rule (reject or accept) is constructed by weighting the evidence of two hypotheses. Third, calibrating the two cost parameters to obtain desired type I error and power makes the decision-based approaches feasible in clinical trial designs, and the robustness of the properties of the designs to cost parameters, irrespective of patient accrual rates, hypotheses, timing of the interim look, and three proposed methods, makes the design very appealing.

Compared with the commonly used Simon's designs, the decision-based Bayesian designs have the following advantages: (1) no need to pause the trial at the end of stage I to wait for all the patients to reach the landmark point; (2) significantly shorter average trial duration and much smaller sample size; (3) a flexible design that could easily add early stopping for efficacy as well as for futility; and (4) reasonable design properties (such as PET), which are based on weight of evidence in the data rather than awkward frequentist hypothesis tests.

In addition, the proposed methods can be easily adapted to randomized trials with survival endpoints. In such trials, the 'difference' in the survival rate between treatment and control groups will be monitored. This 'difference' could be represented as a parameter in the accelerated failure time model or Cox regression model. The terminal decision rule and stopping rule will be constructed using the posterior and predictive distribution of this parameter.

In this paper, we did not include patients that are lost to follow-up or dropout before reaching the landmark point. This information can be easily incorporated into the estimation methods for the Bayesian model [25]. In contrast, designs that use binary endpoints do not have a natural way to incorporate this partial information.

We used quite noninformative prior to reflect limited experience with the experimental regimen under considerations. In certain special situations in which more experience with experimental treatment is available, other priors could be used.

Assuming exponential distributions, trials can be designed with many stages because of the simple sufficient statistics. The grid method using backward induction makes the computation time increase 
linearly with the number of stages. However, the Weibull and grouped-data methods are practically limited to two stages mainly because there are not a small number of sufficient statistics [30,31]. For these two methods, Bayes risk was calculated in a forward fashion, for which the computation time increase exponentially with the number of stages, which makes adding one more interim look computationally infeasible although theoretically possible.

In summary, Bayesian decision-based approaches are feasible in designing clinical trials with appealing properties. As more and more experience accumulates with the application of this approach in real trials, it may be possible to design the trials by directly specifying the costs rather than treating them as tuning parameters.

\section{Acknowledgements}

Many thanks to Frank E. Harrell for his encouragement to initiate this research. The authors gratefully acknowledge the constructive comments of an associate editor and referees.

\section{References}

1. Chan JK, Ueda SM, Sugiyama VE, et al. 578 Analysis of phase II studies on targeted agents and subsequent phase III trials: what are the 579 predictors for success? J Clin Oncol 2008; 26:1511-1518.

2. Korn EL, Arbuck SG, Pluda JM, et al. Clinical trial designs for cytostatic agents: are new approaches needed? J Clin Oncol 2001; 19:265-72.

3. Stone A, Wheeler C, Barge A. Improving the design of phase II trials of cytostatic anticancer agents. Contemporary Clinical Trials 2007; 28:138-145.

4. Panageas KS, Ben-Porat L, Dickler MN, et al. When you look matters: the effect of assessment schedule on progression-free survival. J Natl Cancer Inst 2007; 99:428-32.

5. Gehan EA. The determination of the number of patients required in a preliminary and a follow up 555 trial of a new chemotherapeutic agent. J Chronic Dis 1961; 13:346-353.

6. Fleming TR. One-sample multiple testing procedure for phase II clinical trials. Biometrics 1982; 38(558):143-151.

7. Simon R. Optimal two-stage designs for phase II clinical trials. Controlled Clinical Trials 1989; 10:1-10.

8. Herndon JE. A design alternative for two-stage, phase II, multicenter cancer clinical. Contemporary Clinical Trials 1998; 19(5):440-450.

9. Case LD, Morgan TM. Design of Phase II cancer trials evaluating survival probabilities. BMC Medical Research Methodology 2003; 3:6. DOI: 10.1186/1471-2288-3-6.

10. Lin DY, Shen L, Ying Z, Breslow NE. Group sequential designs for monitoring survival probabilities. Biometrics 1996; 52:1033-1041. DOI: 10.1200/JCO.2009.22.4329.

11. Huang B, Talukder E, Thomas N. Optimal two-stage phase II designs with long-term endpoints. American Statistical Association, Statsitics in Biopharmaceutical Research 2010; 2:51-60. DOI: 10.1198/sbr.2010.09001.

12. Follman DA, Albert PS. Bayesian monitoring of event rates with censored data. Biometrics 1999; 55(2):603-607.

13. Rosner GL. Bayesian monitoring of clinical trials with failure-time endpoint. Biometrics 2005; 61:239-245.

14. Cheung YK, Thall PF. Monitoring the rates of composite events with censored data in phase II clinical trials. Biometrics 2002; 58:89-97.

15. Thall PF, Wooten LH, Tannir NM. Monitoring event times in early phase clinical trials: some practical issues. Clin Trials $2005 ; 2(6): 467-78$.

16. Thall PF, Simon R, Estey EH. Bayesian sequential monitoring designs for single-arm clinical trials with multiple outcomes. Statistics in Medicine 1995; 14:357-379.

17. Lachin J. A review of methods for futility stopping based on conditional power. Statistics in Medicine 2005; 24: 2747-2764. DOI: 10.1002/sim.2151.

18. Pepe MS, Anderson GL. Two-stage experimental designs: early stopping with a negative result. Applied Statistics 1992; 41:181-190.

19. Berry DA. Bayesian clinical trials. Nature Reviews Drug Discovery 2006; 5:27-36. DOI: 10.1038/nrd1927.

20. Staquet MJ, Sylvester RJ. A decision theory approach to phase II clinical trials. Biomedicine 1977; 26(4):262-266.

21. Sylvester RJ, Staquet MJ. Design of phase II clinical trials in cancer using decision theory. Cancer Treatment Report 1980; 64(2-3):519-524.

22. Sylvester RJ. A Bayesian approach to the design of phase II clinical trials. Biometrics 1988; 44(3):823-836.

23. Brunier HC, Whitehead J. Sample sizes for phase II clinical trials derived from Bayesian decision theory. Statistics in Medicine 1994; 13(23-24):2493-2502.

24. Stallard N. Sample size determination for phase II clinical trials based on Bayesian decision theory. Biometrics 1998; 54(1):279-294.

25. Zhao L, Woodworth G. Bayesian decision sequential analysis with survival endpoint in phase II clinical trials. Statistics in Medicine 2009; 28:1339-1352. DOI: 10.1002/sim.3544.

26. Berry SM, Carlin BP, Lee J, Muller P. Bayesian Adaptive Methods for Clinical Trials, Chapman \& Hall/ CRC Biostatistics Series. Boca Raton: CRC Press, 2011.

27. Albert J. Bayesian Computation with R. Springer: New York, 2009. 


\section{Statistics}

28. Kalbfleisch J. Nonparametric Bayesian analysis of survival time data. Journal of the Royal Statistical Society 1978; 40:214-221.

29. Ibrahim JG, Chen MH, Sinha D. Bayesian Survival Analysis. Springer: New York, 2001. 50-53.

30. Muller P, Berry DA, Grieve AP, et al. Simulation-based sequential Bayesian design. Journal of Statistical Planning and Inference 2007; 137(10):3140-3150. DOI: 10.1016/j.jspi.2006.05.021.

31. Brockwell AE, Kadane JB. Sequential analysis by griding sufficient statistics. J. Comput. Graph. Statist 2003; 12:566-584. 